Trauma Berufskrankh 2004 - 6 [Suppl 4] : S471-S478 DOI 10.1007/s10039-003-0810-4

Online publiziert: 28. Januar 2004

(c) Springer-Verlag 2004

M. Herzog • Klinik für Mund-Kiefer-Gesichts-Chirurgie, Unfallkrankenhaus Berlin, Berlin

\title{
Wunden, Narben, Keloide
}

\section{Besonderheiten bei Verletzungen im Gesichtsbereich}

mäßig oberflächlich über den M. masseter, um dann nach Durchtritt durch den M. buccinator in Höhe des 2. oberen Molaren in den Mundvorhof zu münden.

Die Gesichtsweichteile werden von den Knochen des Gesichtsschädels, den Nasenknorpeln und den Zähnen gestützt, diese Strukturen formen das individuelle Gesicht. Die Integrität von Gesichtsschädelskelett und Gesichtsweichteilen ist für die Funktion des binokularen Sehens, des Riechens, der Atmung und des Kauens unerlässlich.

Die mimische Muskulatur inseriert direkt in der Gesichtshaut. Zusätzlich bestehen zahlreiche septale Verbindungen zwischen Gesichtshaut und Muskulatur. Da die mimische Muskulatur sehr unterschiedlich ausgebildet ist, entsteht so der individuelle Gesichtsausdruck.

Die Spannungslinien der Haut (RSTLinien, „relaxed skin tension lines“), die im Wesentlichen im rechten Winkel zur Zugrichtung der mimischen Muskulatur verlaufen und mit zunehmendem Alter den Gesichtsfalten entsprechen, zeigen die Verlaufsrichtung zarter, unauffälliger Narbenbildungen an (• Abb. 1a). Verlaufen Narben senkrecht zu diesen Linien, kann eine breite Narbenbildung nicht ausgeschlossen werden, da die elastischen Kräfte der Haut die Wundränder distrahieren können.

Im Gesicht werden Gesichtsfelder oder ästhetische Einheiten unterschieden (- Abb. 1b). Die Haut dieser Gesichtsfelder ist von unterschiedlicher Dicke und Elastizität, sodass ein Austausch problematisch sein kann. Narben, die an den Grenzen zwischen den Arealen verlaufen, sind meist unauffällig. Narben, die die Gren- zen zwischen Gesichtsarealen kreuzen, können ästhetisch auffällig sein.

Der N. trigeminus innerviert das Gesicht sensibel. Seine Endäste verlassen als N. frontalis, N. infraorbitalis und N. mentalis an den entsprechenden Foramina den Knochen und verlaufen dann oberflächlich unter der Haut. Der N. infraorbitalis wird häufig bei Orbitabodenfrakturen, der N. alveolaris inferior bei Unterkieferfrakturen verletzt.

Der N. facialis verlässt den Schädel am Foramen stylomastoideum und zweigt sich innerhalb der Ohrspeicheldrüse in seine Hauptäste auf. Die Rami zygomatici und buccales laufen nach Verlassen der Glandula parotis von unten an die mimische Muskulatur heran. Sie bilden oft Nervengeflechte und haben eine relativ hohe Regenerationsfähigkeit. Die Rami temporales und der Ramus marginalis regenerieren dagegen sehr viel schlechter. Die Rami temporales verlaufen in der Stirn unter dem M. frontalis, der Ramus marginalis verläuft direkt unter dem Platysma.

Weitere wichtige Strukturen im Gesichtsbereich sind in $\bullet$ Tabelle 1 dargestellt.

\section{Verletzungsformen}

Wunden im Gesichtsbereich treten als isolierte Haut- oder Schleimhautverletzung auf, häufig sind Haut, Muskulatur und Schleimhaut gleichzeitig betroffen. Weichteilverletzungen werden oft in Kombination mit Frakturen des Gesichtsschädels, der Zähne oder anderer Strukturen beobachtet. Weitere Begleitverletzungen betreffen die Schädelbasis, das Gehirn und die Halswirbelsäule, aber auch Nerven, subkutanen Verschiebeschicht, ihr Ausführungsgang zieht zunächst verhältnis- 

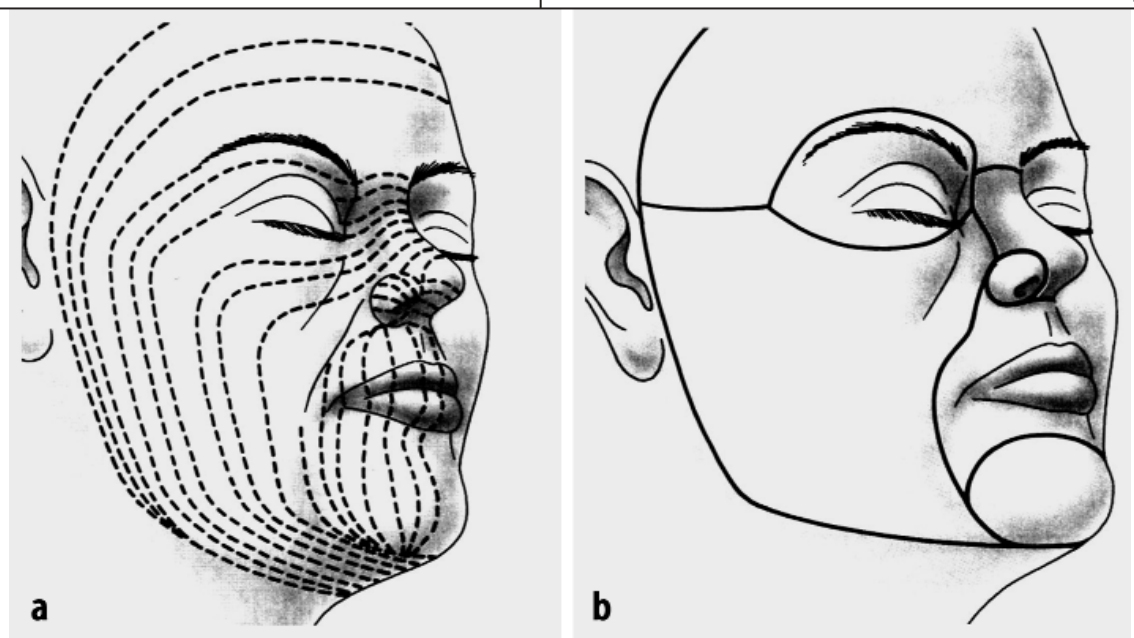

Abb. $1 \Delta$ a RST-Linien („relaxed skin tension lines“). Schmale Narben bei parallelem Verlauf, breite Narben bei Abweichung. $b$ Gliederung des Gesichtes in Felder. In den Grenzlinien zwischen den Arealen verlaufende Narben sind unauffällig

Gefäße, Augen, Tränenwege, Speicheldrüsen und ihre Ausführungsgänge, Nase und Zähne.

\section{Knöcherne Verletzungen}

Knöcherne Verletzungen betreffen das Mittelgesicht und den Unterkiefer. Das Mittelgesicht reicht von den Zähnen bzw. dem Alveolarfortsatz des Oberkiefers bis zur Schädelbasis. Es umfasst den Oberkiefer, die Siebbeine, Jochbeine, Tränenbeine, Keilbeine und den Vomer. Für seinen Aufbau ist ein kompliziertes Höhlensystem kennzeichnend (Augenhöhle, Nasenhaupt- und Nasennebenhöhlen). Da bei Frakturen regelmäßig die Schleimhautauskleidung der Nebenhöhlen einreißt und es bei Frakturen zahntragender Kieferabschnitte über den Parodontalspalt zu einer Verbindung zwischen Mundhöhle und Knochenmark kommt, sind diese Frakturen als offene Frakturen anzusehen.

\section{Zahnverletzungen}

Zähne sind sehr häufig Traumen ausgesetzt und werden dabei unterschiedlich stark verletzt. Es werden Zahnfrakturen und Zahnluxationen unterschieden, beide Verletzungen können gleichzeitig auftreten. Der Zahnschmelz ist reparationsund regenerationsunfähig, Dentin kann mit der Bildung von Sekundärdentin auf eine Verletzung reagieren. Das Zahnmark (Pulpa) ist nach Abschluss des Wurzelwachstums bei offenen Verletzungen nicht regenerationsfähig und nicht vital zu erhalten. Kommt es bei Luxation von Zähnen zu einer Verletzung der Wurzelhaut, ist die Einheilung entscheidend behindert. Bestenfalls kommt es zu einer Verfestigung des Zahnes in der Alveole durch ankylotische Vorgänge. Nach unterschiedlichen Zeiträumen werden solche Zähne resorbiert.

Zum Ausschluss von knöchernen Verletzungen bzw. zum Erkennen von Fremdkörpern ist der Einsatz bildgebender Verfahren unerlässlich. Bei Anwendung konventioneller Aufnahmen ist die Darstellung aller betroffenen Skelettabschnitte in 2 senkrecht aufeinander stehenden Ebenen erforderlich. Heute kommt die Computertomographie zunehmend zur Anwendung. Insbesondere zum Erkennen nicht röntgendichter Strukturen, wie Holz, kann der Einsatz der Kernspintomographie und der Sonographie zweckmäßig sein.

Wunden im Gesichtbereich sind häufig kontaminiert. Neben der exogenen Kontamination mit Schmutz, Fremdkörpern oder auch Chemikalien ist v. a. an die Kontamination durch Speichel, Zahnfragmente, Füllungsfragmente und Zahnersatzteile zu denken.

\section{Primärversorgung}

Die Mund-Kiefer-Gesichts-Region zählt dank ihrer guten Durchblutung zu den Körperregionen mit überdurchschnittlich guter Heilungstendenz. Dies schlägt sich in einigen $z$. T. von denen der allgemei- nen Chirurgie abweichenden Regeln der Wundversorgung nieder. So findet z. B. die 6-h-Marke als Grenze für die primäre Naht oder die Osteosynthese keine Anwendung. Eine aufgeschobene Primärversorgung nach $12-24 \mathrm{~h}$, in Ausnahmefällen auch nach mehreren Tagen, ist zulässig.

Die genaue Übersicht über das Ausmaß des Weichteilschadens und eines eventuellen Gewebeverlustes ist bei der frischen Verletzung eher möglich als nach Abheilung mit narbigen Verziehungen. Daher erfolgt bei allen Weichteilverletzungen eine sorgfältige Wundinspektion, bei der alle Wunden bis zu ihrem tiefsten Punkt dargestellt werden. Stark blutende Gefäßverletzungen werden dabei durch Unterbindung, Umstechung oder Koagulation versorgt. Eine mikrochirurgische Gefäßrekonstruktion ist wegen der guten Kollateralenbildung im Gesicht in der Regel nicht erforderlich. Nasenseptum- und Ohrhämatome werden entlastet und drainiert, um Knorpelnekrosen zu vermeiden.

Liegen gleichzeitig knöcherne Verletzungen oder Zahnluxationen vor, so sind diese zunächst zu reponieren und in regelrechter Okklusion und Fragmentstellung endgültig mit Osteosynthesen und Schienen stabil zu versorgen. Dabei ist besonderer Wert auf die regelrechte Okklusion, den korrekten Zusammenbiss der Zähne, zu legen. Bereits Verschiebungen von Bruchteilen eines Millimeters können zu erheblichen Störungen des stomatognathen Systems führen und schwer behandelbare Schmerzzustände verursachen.

Vollständig luxierte Zähne werden in Kochsalz- oder Ringerlösung aufbewahrt, da der Wurzelzement bereits nach $30 \mathrm{~min}$ Trockenheit irreversible Schäden aufweist und eine physiologische Einheilung eines so geschädigten Zahnes nicht mehr möglich ist.

Erst anschließend erfolgt ggf. die mikrochirurgische Rekonstruktion von Nerven oder von Ausführungsgängen der Speicheldrüsen. Nach Wiederherstellung der die Gesichtskonturen bestimmenden und die Gesichtsweichteile stützenden Gesichtsschädelknochen werden Schleimhaut, Muskulatur und Haut versorgt, da so die Gewebe weniger traumatisiert werden und günstigere Ausheilungszustän- 


\begin{tabular}{|l|}
\hline Tabelle 1 \\
\hline Besondere Strukturen des Gesichts \\
\hline Mimische Muskulatur \\
\hline Lippen \\
\hline Zunge \\
\hline Nase \\
\hline Lider \\
\hline Augen \\
\hline Tränenwege \\
\hline Ohrmuschel \\
\hline Gesichtsschädel \\
\hline Zähne \\
\hline Nerven \\
N.trigeminus \\
N.facialis \\
N.hypoglossus \\
\hline Gefäße \\
A.facialis \\
A.temporalis \\
A.maxillaris \\
\hline Speicheldrüsen \\
Glandula parotis \\
Glandula submandibularis \\
Glandula sublingualis \\
\hline Knorpel \\
Nasenknorpel \\
Ohrknorpel \\
Tarsus \\
\hline
\end{tabular}

de zu erwarten sind. Oberstes Prinzip ist dabei die Versorgung von innen nach außen. Die besten Ergebnisse können erreicht werden, wenn die gesamte Versorgung in einer einzigen Operation gelingt. Ausnahmen sind nur bei lebensbedrohlich verletzten polytraumatisierten $\mathrm{Pa}$ tienten zulässig, bei denen der für eine definitive Versorgung notwendige Zeitaufwand nicht zu rechtfertigen ist.

Bei temporärer Versorgung (Polytrauma) werden die Wunden nach Blutstillung, Fremdkörperentfernung und schonender Wundreinigung locker adaptiert und ggf. drainiert. Nervenstümpfe werden an beiden Enden durch nichtresorbierbares Nahtmaterial markiert.

Teilamputierte Weichteile können unter Verwendung mikrochirurgischer Techniken revaskularisiert [12] werden. Vollständig traumatisch amputierte Weichteile sind unter folgenden Bedingungen [15] replantationsfähig:

- Ischämiezeit: maximal 12-16 h,
Trauma Berufskrankh $2004 \cdot 6$ [Suppl 4] : S471-S478

DOI 10.1007/s10039-003-0810-4

C) Springer-Verlag 2004

\section{Herzog}

\section{Wunden, Narben, Keloide. Besonderheiten bei Verletzungen im Gesichtsbereich}

\section{Zusammenfassung}

Wunden im Gesichtsbereich beeinträchtigen die Funktion und Ästhetik und haben daher einen besonderen Stellenwert für Patient und Arzt. Sie treten als isolierte Haut- oder Schleimhautverletzung auf. Häufig sind Haut, Muskulatur und Schleimhaut gleichzeitig betroffen. Weichteilverletzungen werden oft in Kombination mit Frakturen des Gesichtsschädels, aber auch bei Mehrfachverletzungen beobachtet. Wegen der guten Durchblutung des Gesichtsbereichs ist eine Primärversorgung fast immer möglich, auch wenn die Wunden durch die Beteiligung der Mundhöhle häufig kontaminiert sind. Bei ihrer Versor-

gung gilt das Prinzip: von innen nach außen. Zunächst werden knöcherne Verletzungen, anschließend Schleimhaut und Muskulatur und erst abschließend die Haut versorgt. Bissverletzungen können in der Regel nach den gleichen Kriterien behandelt werden. Narben verursachen insbesondere um Mund, Augen und Nase erhebliche funktionelle Störungen. In der Regel erfolgt die Korrektur frühestens nach 6 Monaten. Die Behandlung von Keloiden ist problematisch.

\section{Schlüsserwörter}

Wunden · Narben · Keloide · Gesichtsbereich

\section{Wounds, scars, keloids. Characteristics of facial injuries}

\section{Abstract}

Facial injuries affect function and esthetics and play an important role in the opinion of patient and physician. Facial injuries are found as isolated wounds of the soft tissues, in many cases in combination with injuries of the facial skeleton, of the skull base, or other injuries. Vascularization of the face is excellent, enabling wound closure in most cases, even in contaminated injuries. The following procedure should be used: (1) reposition and stabilization of fractures of the facial skeleton, (2) reconstruction of soft tissues, and (3) suture of the skin. In most cases, dog bite wounds are managed in the same way. Scars of the lips, the nose, and the eyelids cause functional obstruction. Corrections can be done 6 months after mutilation. Treatment of keloids is questionable.

\section{Keywords}

Wounds · Scars · Keloids · Facial injury 


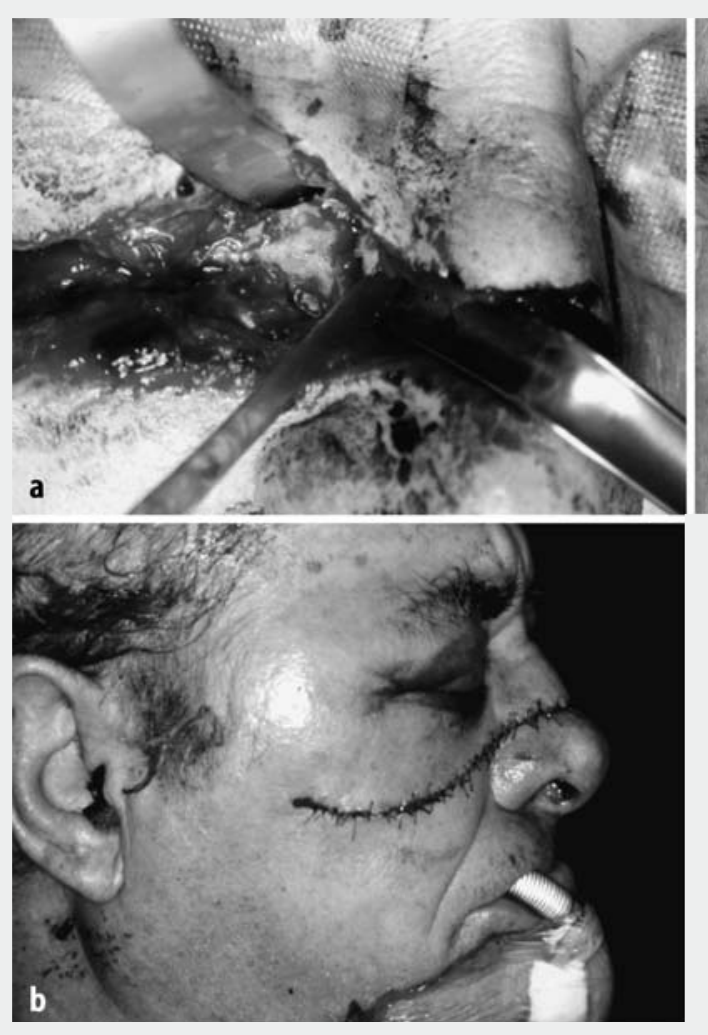

Abb. $2<$ Kettensägenverletzung des Gesichtes. a Ausgedehnte Zerreißungen von Weichteilen der Nase und der Wange mit Beteiligung des Knochens. $b$ Nach primärer Wundversorgung. $c$ Befund 1 1/2 Jahr nach dem Unfall
- Grad der Zertrümmerung und Quetschung: gering,

- anastomosefähige Gefäße: vorhanden,

- Nervenanastomosen: möglich.

Bei Fehlen dieser Grundvoraussetzungen sind als alternative Techniken die Verfahren der plastisch-rekonstruktiven Chirurgie anzuwenden. Dies gilt besonders für traumatische Amputationen durch Bissverletzungen, bei denen Misserfolge gehäuft beobachtet [11] werden.

Obwohl Gesichtswunden in einem hohen Prozentsatz kontaminiert sind, ist eine radikale Wundexzision in der Technik nach Friedrich meistens nicht erforderlich. Gelegenheitswunden können noch nach 12$24 \mathrm{~h}$, in Ausnahmefällen auch noch nach $48 \mathrm{~h}$ primär versorgt werden. Nekrotische oder schmutztätowierte Gewebeabschnitte werden sparsam entfernt, Wundtaschen werden vollständig dargestellt. Dies gilt besonders für den Bereich der Augenlider, der Nase oder der Lippenrot-WeißGrenze. Hier sollte versucht werden, auch kleine oder schlechternährte Haut-, Schleimhaut- oder Knorpelteile im Sinne eines freien Transplantates erhalten, da sie für die normale Form und Funktion unverzichtbar und sekundär nur sehr schwierig zu rekonstruieren sind ( $\bullet$ Abb. $2 a-c)$.

Nur bei ausgedehnten Quetschwunden (Pferdebiss), Explosions-, Schuss-, Säureoder Laugenverletzungen kann die Schaffung glatter unversehrter Wundränder über den Weg eines radikalen Débridements erforderlich werden. Sofern es der Allgemeinzustand erlaubt und kein Anhalt für eine fortschreitende Entzündung besteht, kann abgewartet werden, bis eine eindeutige Demarkierung des nekrotischen Gewebes zu erkennen ist. Die Wunden werden zunächst dräniert und mit feuchten antiseptischen Verbänden (Rivanol ${ }^{\circledR}$, Braunol $\left.^{\circledR}\right)$ abgedeckt. Der dann unvermeidliche Gewebeverlust zieht die Notwendigkeit plastisch-chirurgischer Maßnahmen nach sich.

\section{Tetanusschutz}

Auch wenn nur intraorale oder intranasale Verletzungen vorliegen, ist der Tetanusschutz zu überprüfen und ggf. aufzufrischen.

\section{Antibiotikaprophylaxe}

Nahezu jede Gesichtswunde mit Beteiligung der Mund- bzw. der Nasenhöhle wird durch die Wundsetzung selbst oder sekundäre Keimeinwanderung kontaminiert. Oberflächliche, klinisch saubere Haut- und Schleimhautverletzungen erfordern keine Antibiotikagabe. Bei ausgedehnten Weichteilverletzungen, insbesondere bei gleichzeitigem Vorliegen knöcherner Verletzungen, hat sich die perioperative intravenöse einmalige Gabe eines Breitbandantibiotikums bewährt (Amoxicillin/Clavulansäure, Ampicillin/ Sulbactam, Clindamycin, Cephalosporine; [5]).

\section{Operatives Vorgehen}

Bagatell- und weniger ausgedehnte Weichteilverletzungen können in Leitungs- und Lokalanästhesie versorgt werden. Dabei sind Leitungsanästhesien an den typischen Nervenaustrittspunkten zu bevorzugen. Es finden $2 \%$ - oder 3\%-Zubereitungen der Lokalanästhetika mit einem Adrenalinzusatz von 1:100.00o Verwendung. Ausgedehnte Weichteilverletzungen und Verletzungen mit Gesichtsschädelfrakturen werden bevorzugt in Allgemeinnarkose versorgt, da infolge der langen Operationsdauer nur so die erforderliche Ruhe des Patienten bei gleichzeitiger Relaxation des Operationsgebietes gewährleistet sind. Um ggf. die Okklussion beurteilen zu können, wird eine nasale Intubation oder eine submandibuläre Ausleitung erforderlich. Eine Tracheotomie erfolgt bei absehbarer Langzeitbeatmung.

Die Versorgung von Gesichtsverletzungen erfolgt mit einem feinen Instrumentarium, ggf. unter Lupenvergrößerung. Bei Lidverletzungen ist ein Operationsmikroskop hilfreich, bei der mikrochirurgischen Versorgung von Nervenverletzungen unabdingbar. Alle erkennbaren Strukturen werden schichtweise rekonstruiert. Besonderes Augenmerk ist auf funktionell und ästhetisch kritische Zonen wie die Augenbrauen, die Lidkanten, die Nasenflügel, den Mundwinkel und die Lippenrot-Weiß-Grenze zu legen. Mit Ausnahme oberflächlicher Bagatellverletzungen werden alle Wunden dräniert. Dabei ist bei tieferen Verletzungen eine Re- 
Tabelle 2

Versorgungsschema bei Weichteilwunden. (Nach [15])

\begin{tabular}{|c|c|c|c|}
\hline Art der Verletzung & Nahttechnik & Material & $\begin{array}{l}\text { Nahtentfernung } \\
\text { (Tage post Op.) }\end{array}$ \\
\hline Komplizierte Wunde mit Knochenbeteiligung & Schichtweise & & \\
\hline Periost & & $3 \times 0,4 \times 0$ resorbierbar & - \\
\hline Muskulatur & & $3 \times 0-5 \times 0$ resorbierbar & - \\
\hline Haut & Einzelknopfnaht & $5 \times 0,6 \times 0$ nichtresorbierbar & 5 \\
\hline Haut & Intrakutan & $4 \times 0,5 \times 0$ nichtresorbierbar & $10-15$ \\
\hline \multicolumn{4}{|l|}{ Speicheldrüsen } \\
\hline Parenchym & Kapselnaht & $3 \times 0,4 \times 0$ resorbierbar & - \\
\hline Gang & End zu End (Mikroskop, Lupenbrille) & $7 \times 0-9 \times 0$ nichtresorbierbar & - \\
\hline Nerven und Gefäße & Mikrochirurgisch & $\leqq 9 \times 0$ nichtresorbierbar & - \\
\hline
\end{tabular}

don-Saugdränage angezeigt. Bei eher oberflächlichen Verletzungen kommen feine Silastikröhrchen zum Einsatz, die nach 24 h entfernt werden.

Besonders entstellende Narben entstehen, wenn es zum Einheilen von Schmutzeinsprengungen kommt, die zu kaum korrigierbaren Schmutztätowierungen führen. Daher werden alle flächenhaften Schürfwunden des Gesichts mit Schmutzeinsprengungen ausgebürstet, um so die Schmutzpartikel aus der Wunde zu entfernen. Als Spülflüssigkeit während der Bürstenbehandlung können physiologische Kochsalzlösung, Polyvidonjod oder auch 1\%ige Wassersuperoxydlösung verwendet werden. Eine Alternative, insbesondere bei ausgedehnten Verletzungen und aggressiven Fremdkörpern (Mörtel, Beton) stellt die Jet-Lavage dar [6].

Die Versorgung von Schleimhautverletzungen erfolgt mit Einzelknopfnähten $(3 \times 0,4 \times 0)$ mit nichtresorbierbarem Material (Polyester) oder Polylactin (Vicryl ${ }^{\circledR}$ ). Letzteres ist auch nach 10 Tagen nicht aufgelöst, sodass es dann entfernt werden sollte.

Für die Naht der Muskulatur empfiehlt sich feines resorbierbares Material der Stärken $3 \times 0$ oder $4 \times 0$, im direkten subkutanen Bereich oder im Bereich der Lider auch der Stärke $5 \times 0$.

Die Hautränder werden entweder mit 4×0- oder 5×0-Intrakutannähten oder mit monofilem $6 \times 0-N a h t m a t e r i a l$ in Einzelknopftechnik oder in fortlaufender Naht adaptiert, im Lidbereich auch $7 \times 0$. Einzelfäden bzw. fortlaufende Nähte werden am 5. postoperativen Tag entfernt, um ein Einwachsen des Epithels entlang der Fä- den und damit störende Narbenbildungen zu vermeiden. Intrakutane Fäden können 10-14 Tage belassen werden (• Tabelle 2). Ein stützender Pflasterverband (Steristrip) kann Hautspannungen auffangen.

\section{Zungenverletzungen}

Oberflächliche Zungenverletzungen werden einschichtig durch Naht versorgt. Bei tiefen Zungenverletzungen ist eine mehrschichtige Versorgung zu empfehlen, blutende Zungengefäße sind sorgfältig zu unterbinden oder zu koagulieren, da sich bei Nachblutungen lebensbedrohende Hämatome entwickeln können.

\section{Bissverletzungen}

Bei Bissverletzungen handelt es sich v. a. um Hundebisse, seltener um Katzen- oder Menschenbisse. Sie betreffen v. a. Lippen, Kinn, Ohren und Nase. Kinder sind überproportional häufig betroffen [17]. Bissverletzungen sind regelmäßig als infiziert anzusehen. Bei allen Tierbissen ist eine Tollwutabklärung erforderlich.

Die Behandlung von Bissverletzungen wird kontrovers diskutiert, wobei im Gesichtsbereich die Vermeidung einer Wundinfektion mit einem guten ästhetischen und funktionellen Ergebnis konkurriert. Nach eigenen Erfahrungen an über 150 Hundebissverletzungen und in Übereinstimmung mit der Literatur [18] können diese in der Regel primär innerhalb der ersten $6 \mathrm{~h}$ versorgt werden. Nach genauer Inspektion, wobei auch bei kleinen Hautverletzungen (Katzenbiss) nach tiefen Wundtaschen, schlecht durch- bluteten Arealen und Fremdkörpern (Zahnstein!) zu fahnden ist, erfolgen eine sparsame Begradigung von Wundrändern und eine zurückhaltende Entfernung von schlecht durchbluteten Gewebeanteilen. Ein schichtweiser Wundverschluss schließt sich an. Eine ausreichende Dränage ist sicherzustellen.

Bei oberflächlichen Haut- oder Schleimhautverletzungen erfolgt eine einmalige perioperative Antibiotikagabe, bei tieferen Verletzungen oder bei längerem Intervall zwischen Trauma und Versorgung eine Antibiotikatherapie für 5 Tage. Als Antibiotika sind Amoxycillin/Sulbactam bzw. Amoxycillin/Clavulansäure, Cephalosporine der 3. Generation oder Clindamycin $[5,11,17]$ besonders geeignet.

Bei immunsuprimierten Patienten, schlecht eingestellten Diabetikern und Alkoholikern treten Wundinfektionen häufiger auf [3], engmaschige Überwachung, evtl. sogar der Verzicht auf einen sofortigen dichten Wundverschluss ist daher angezeigt. Es erfolgt eine Antibiotikatherapie von wenigstens 5 Tagen.

Bereits infizierte Bissverletzungen erfordern eine andere Vorgehensweise. Die Wunden werden bis an den tiefsten Punkt exploriert, nekrotisches Gewebe wird entfernt. Es erfolgt zunächst eine offene Wundbehandlung mit Einlage von Gummidränagen oder antiseptischen Tamponaden und Verbänden (Rivanol ${ }^{\circledR}$, Braunol ${ }^{\circledR}$ ), extra-intraorale Dränagen sind zu vermeiden. Eine Antibiotikatherapie ist obligatorisch, ein Wundabstrich und eine Resistenzbestimmung oft zu empfehlen. Nach Reinigung der Wunden und Abklingen der Allgemeinsymptome erfolgt eine 




Abb. $3<$ Ausgedehnte Narben im Bereich der linken Orbita mit erheblicher Funktionsstörung der Lider



Abb. $4 \nabla$ Nicht versorgte, 6 Wochen alte Riss-Quetsch-Verletzung des linken Mundwinkels. a Befund präoperativ. $b$ Exzision von Nekrosen, Darstellung der Muskelstümpfe. c Befriedigendes ästhetisches Ergebnis. $d$ Befriedigendes funktionelles Ergebnis
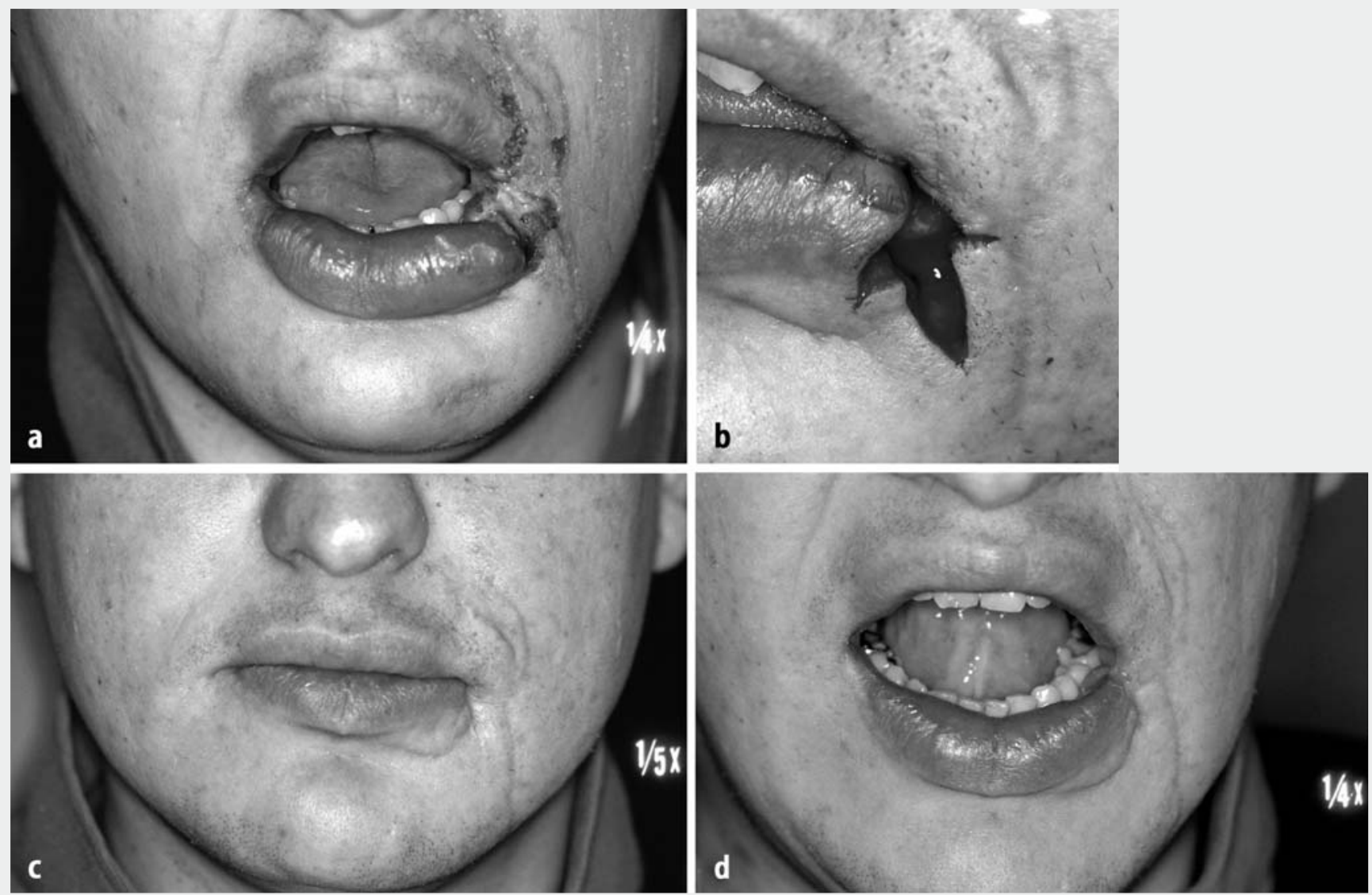

lockere Sekundärnaht mit ausreichender Dränage.

\section{Sekundärbehandlung - Narben und Keloide}

Die normale Wundheilung verläuft über die Fibroblastenaktivierung, die die Synthese der kollagenen Fasern induziert. Die Wachstumsfaktoren PDGF $\alpha$ und PDGF $\beta$ steuern diese Synthese. Das Narbengewebe zeigt meist ein gerichtetes Faserbün- del. Kommt es bei der Kollagensynthese (Helixketten, Superhelixketten, Super-Super-Helix mit alternierender Drehung) zu einer Störung dieser Ordnung, ist eine Dehiszenz der Narbe zu erwarten [15].

Der Faserverlauf des Kollagens wird durch die Lastaufnahme bestimmt. Eine zu frühe Belastung kann zu einem Bruch des vernetzten Systems führen. Die Stabilität der Narbe hängt dabei immer von der Fasergeometrie ab und erreicht in der
Haut nach 4 Wochen erst 20\% der ursprünglichen Gewebefestigkeit.

Die Haut des Erwachsenen enthält $85 \%$ Typ-I-Kollagen (besteht aus $\alpha_{1}-(\mathrm{I})_{2}-$ $\alpha_{2}$-Peptidketten und 15\% Typ-III-Kollagen (besteht aus $\alpha_{1}$-(III) ${ }_{3}$-Peptidketten. Epidermis und Dermis zeigen dabei eine gleichartige Typ-I-Verteilung, hingegen nimmt Typ III zur Tiefe hin ab [15].

Bei Keloiden findet sich eine etwa 2ofache Steigerung der Kollagensynthese. Die stimulierenden multifaktoriellen Reize 

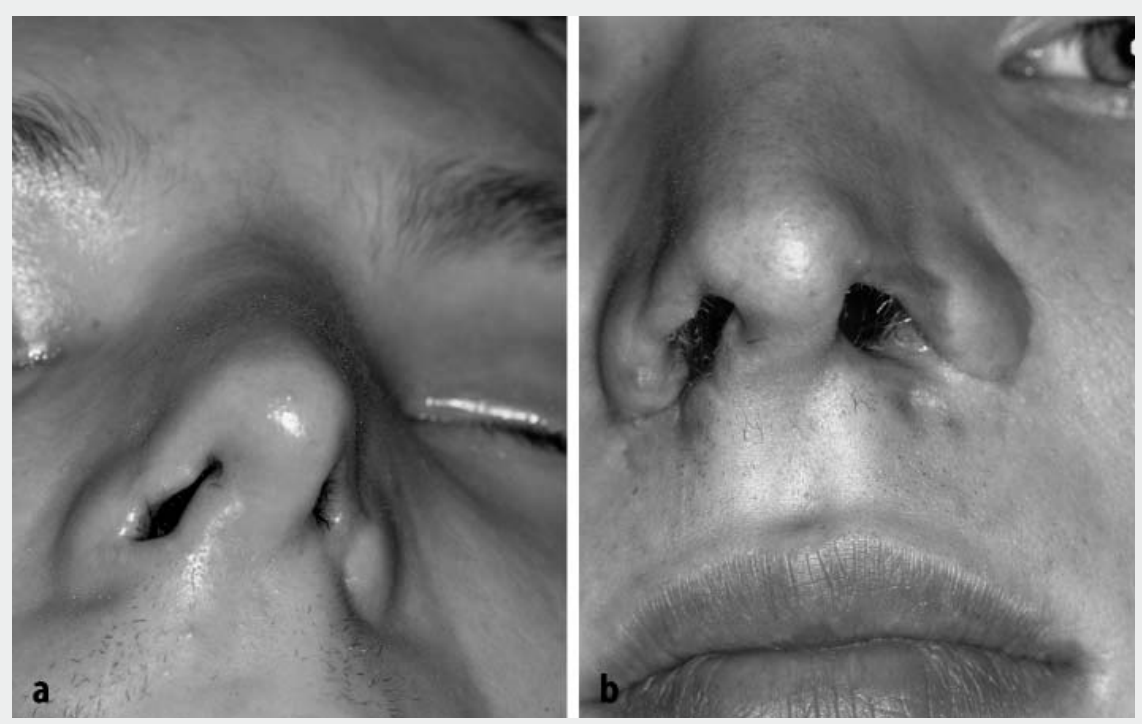

Abb. $5<$ Ausgedehnte Knorpelnekrosen im Bereich der Nase nach Langzeittamponade wegen ebensbedrohender Blutungen. a Kollaps der $\mathrm{Na}$ senflügel mit Behinderung der Nasenatmung. b Befund nach Rekonstruktion der Nasenknorpel durch Knorpeltransplantat

Abb. $6 \nabla$ Durch belassenen Fremdkörper bedingte Narbe im Unterlippen- und Wangenbereich. a Ausgedehnte auffällige schmerzhafte Narbe. b Ursächlicher Glassplitter. c Befund 6 Monate nach Revision
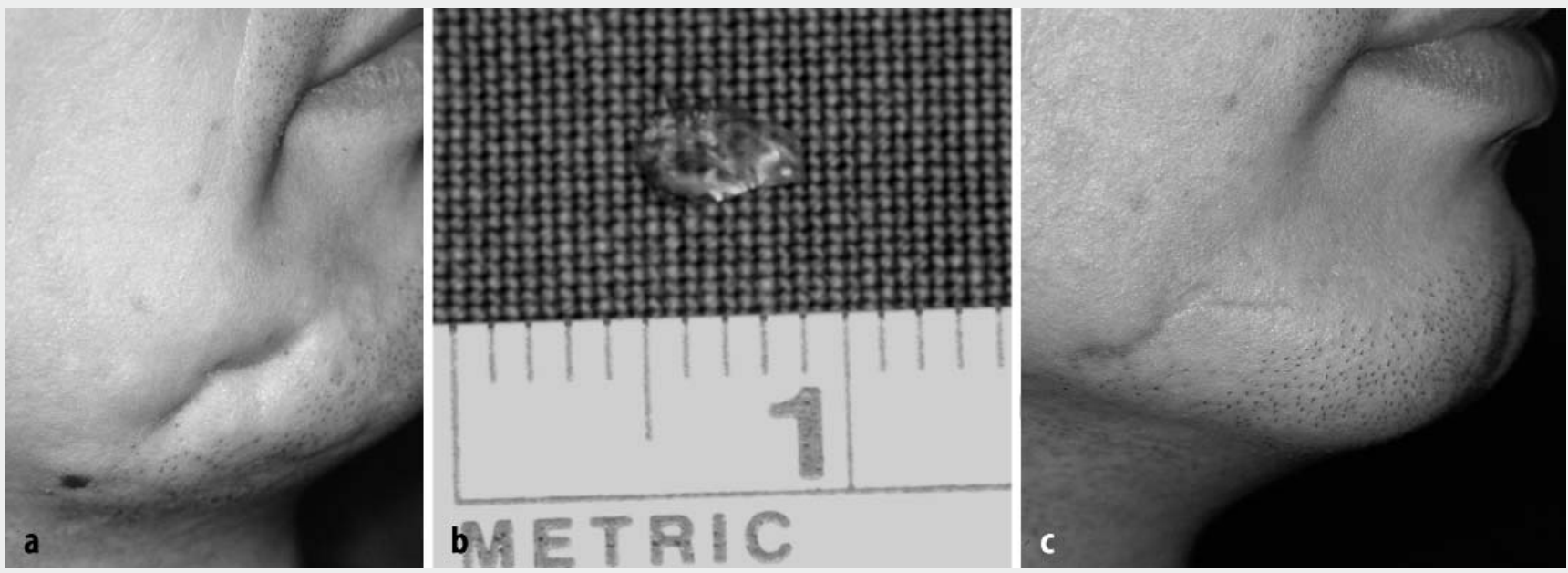

sollen über das Immunsystem einwirken (Autoantigene?). Morphologisch sind neben den ungeordneten verzweigten kollagenen Septen reichlich Mukopolysaccharide eingelagert. In frühen Keloiden findet man häufig Typ-III-Kollagen, während sog. reife Keloide (ca. 1 Jahr bestehend) mehr Typ-I-Kollagen enthalten. Diese Unterschiede legen die Annahme eines Kodierungsfehlers in der Kollagensynthese bei Keloiden nahe [15]. Für diese Annahme spricht auch eine familiäre Häufung. Als weitere Faktoren der Ätiopathogenese der Keloide können allergisch-hyperergische Phänomene, bakterielle Reize, Spannung und Traumatisierung sowie Fremdkörper (Sand, Glas) angesehen werden. Im Tierexperiment konnte bislang kein Keloidmodell nachgewiesen werden [10].
Keloidale Narben zeichnen sich neben dem Gewebeüberschuss dadurch aus, dass sie tumorartig das Gebiet der eigentlichen Narbe verlassen und auf primär unauffälliges, gesundes Gewebe übergreifen. Neben der ästhetischen Beeinträchtigung verursachen Keloide Juckreiz, Spannung, Rötung, Schmerzen und Funktionsstörungen.

Insbesondere um Mund, Augen und Nase verursachen Narbenzüge erhebliche funktionelle Störungen. Ektropionierungen des Unterlides gehen mit Tränenfluss und rezidivierender Konjunktivitis (- Abb. 3), Ektropionierungen der Lippen mit Speichelinkontinenz und Störungen der Aussprache einher (• Abb. 4a-d), Narbenzüge im Wangenbereich rufen Mundöffnungsbehinderungen und Bissverletzungen hervor. Durch Narben im Bereich der Nasenflügel kommt es zum Kollaps der Nasenklappe und damit zu schwer- wiegenden Atmungsbehinderungen und Belüftungsstörungen der Nasennebenhöhlen (• Abb. 5a,b).

Sind nach einer Gesichtsverletzung auffällige Narben verblieben, kann nach Ausnutzen der konservativen Möglichkeiten mit Kompressionsverbänden, Pflastern und Massagen die operative Narbenkorrektur einen wesentlichen Gewinn an Funktion und Ästhetik bedeuten. Sie sollte frühestens 6, besser erst 12 Monate nach der Verletzung durchgeführt werden, wenn die Narbenschrumpfung weitgehend abgeschlossen ist. Nur ausnahmsweise bei erheblichen Stufenbildungen und funktionellen Beeinträchtigungen ist eine Korrektur zu einem früheren Zeitpunkt angezeigt (• Abb. 6a-c).

Kleine Stufen innerhalb der Haut oder geringe Hypertrophien werden durch hochtourige Dermabrasion mit diaman- 
tierten Schleifkörpern abgetragen. Alternativ können hierzu auch verschiedene Lasersysteme wie der $\mathrm{CO}_{2}$-Laser oder der Erbium:Yag-Laser eingesetzt werden [8]. Jede Narbenkorrektur ist sorgfältig zu planen. Es ist zu prüfen, welche der betroffenen Gewebeschichten ursächlich für Verziehungen oder Stufenbildungen ist. Gegebenenfalls wird die Revision der ganzen Narbe mit Darstellung aller beteiligten Schichten notwendig. Dies gilt besonders bei Stufen der Lippenrot-WeißGrenze, des Naseneingangs und der Lidkanten.

Die Schnittführung wird dabei noch vor der Lokalanästhesie aufgezeichnet. Der Narbenverlauf sollte so abgewandelt werden, dass er sich möglichst optimal in die mimische Faltenbildung einfügt (RSTLinien, Gesichtsfelder). Die Z-Plastik, der Austausch von an den Wundrändern gestielten, mehr oder weniger spitzwinkligen Dreiecken, ist eine meistens geeignete Maßnahme. Zugleich wirkt eine Z-Plastik der bei jeder Heilung auftretenden Verkürzung der Narbenstrecke entgegen.

Der Austausch größerer Dreiecke bietet sich immer dann an, wenn mit ihnen der neue Narbenverlauf in langgestreckte mimische Falten, z. B. in die Nasolabialfalte, verlagert werden kann. Dagegen eignen sich zahlreiche kleine Dreiecke besser zur Korrektur großer Narben in glatten Flächen wie der Wangen. Neben der Z-Plastik kommen auch andere Nahlappenplastiken zur Anwendung.

In Fehlstellung verheilte Frakturen, Knorpel- oder Knochendefekte, ein Enophthalmus oder Bulbustiefstand werden vorher, spätestens im Rahmen der Narbenkorrektur behoben. Ein nicht korrekt eingestellter Diabetes mellitus oder eine floride Akne stellen eine Kontraindikation für eine Narbenkorrektur dar.

Ablederungsverletzungen hinterlassen flächige, höckrige Narben, die im Gesicht neben Funktionsstörungen auch Entstellungen verursachen. Nach Exzision kann der Hautersatz mit freien autogenen Transplantaten erfolgen (retroaurikulär, Fossa supraclavicularis). Textur und Kolorit des Spenderareals differieren jedoch meist stark von dem des Empfängerareals, sodass ein Hautersatz mit gestielten Nahlappen zu bevorzugen ist. Der Entnahmebereich kann durch Gewebeexpander vor- gedehnt werden sodass ein primärer, spannungsfreier Verschluss des Entnahmebezirkes möglich wird. Das so gewonnene Gewebe entspricht in seinen Eigenschaften weitgehend dem des Empfängerareals [2].

Die Erwartungshaltung bei Narbenkorrekturen ist hoch. Viele Patienten hoffen auf eine vollständige Beseitigung der Narben. Es ist daher rechtzeitig darauf hinzuweisen, dass dies nicht möglich ist und dass das Ziel einer Narbenkorrektur darin besteht, funktionell und ästhetisch störende Narben unauffälliger zu gestalten.

Die Behandlung von Keloiden ist problematisch. Rezidive treten häufig auf, eine kausale Behandlung ist nicht bekannt. Atraumatische Exzisionen, spannungsfreie Naht mit anschließender Kompression und lokale physikalische Maßnahmen in Form von speziellen Pflasterverbänden [1] unterstützen die Behandlung. Die intrakutane Injektion von Triamcinolon kann hilfreich sein. Beim Erwachsenen wird über gute Ergebnisse nach Anwendung ionisierender Strahlen [9] berichtet.

\section{Korrespondierender Autor Prof.Dr. Dr. M. Herzog}

Klinik für Mund-Kiefer-Gesichts-Chirurgie, Unfallkrankenhaus Berlin, Warener Straße 7, 12683 Berlin, E-mail:michael.herzog@ukb.de

Interessenkonflikt: Keine Angaben

\section{Literatur}

1. Bradford BA, Breault LG, Schneid T, Englemeier RL (1999) Silicone thermoplastic sheeting for treatment of facial scars: an improved technique.J Prosthodont 8 : 138-141

2. Breier T, Becker R (1992) Die kontrollierte Weichgewebedehnung im Kopf-Hals-Bereich. Dtsch Z Mund Kiefer Gesichtschir 16: 296-300

3. Callaham M (1980) Dog bite wounds. JAMA 244: 2327-2328

4. Gassner R, Tuli T, Hächl O, Rudisch A, Ulmer H (2003) Cranio-maxillofacial trauma: a 10 year review of 9543 cases with 21067 injuries.J Craniomaxillofac Surg 31: 51-61

5. Gerlach KL, Pape HD (1988) Untersuchungen zur Antibiotikaprophylaxe bei der operativen Behandlung von Unterkieferfrakturen.Dtsch Z Mund Kiefer Gesichtschir 12: $497-500$

6. Gross A, Cutright DE, Bhaskar SN (1971) The effects of antibiotics and pulsating water jet lavage on contaminated wounds. Oral Surg Oral Med Oral Pathol 31: $32-38$
7. Heeg P,Weingart D (2000) Hygiene und Infektionsprävention. In: Schwenzer N, Ehrenfeld M (Hrsg) Allgemeine Chirurgie Bd 1. Thieme, Stuttgart S 72

8. Landthaler M, Hohenleutner U (1993) Lasertherapie von Hautveränderungen im Gesicht und an der Mundschleimhaut. Fortschr Kiefer Gesichtschir 38: 143-145

9. Maarouf M, Schleicher U, Schmachtenberg A, Ammon J (2002) Radiotherapy in the management of keloids. Clinical experience with electron beam irradiation and comparison with X-ray therapy. Strahlenther Onkol 178: 330-335

10. Polo M, Kim YJ, Kucukcelebi A et al. (1998) An in vivo model of human proliferative scar.J Surg Res 74: 187195

11. Rettinger G, Reichensperger-Goertzen C (1995) Gesichtsverletzungen durch Hundebiss. HNO 43: 159164

12. Reuther JF (1990) Historische Entwicklung und derzeitiger Stand der Mikrogefäßchirurgie. Fortschr Kiefer Gesichtschir 39: 2-7

13. Rusch MD Grunert BK, Sanger JR, Dzierzynski WW, Matloub E (2000) Psychological adjustment in children after traumatic disfiguring injuries. A 12 month follow up. Plast Reconstr Surg 106: 1451-1458

14. Schubert J, Maurer P, Wilhelms D, Socolowsky S (1995) Erfahrungen mit Cefitiam als perioperative Antibiotikaprophylaxe in der MKG-Chirurgie.Dtsch Z Mund Kiefer Gesichtschir 19: 19-21

15. Schumann D, Hyckel P (1997) Weichteilverletzungen, Verbrennungen und Narben. Mund Kiefer Gesichtschir 1 [Suppl 1]: 50-57

16. Sclafani AP, Gordon L, Chadha M, Romo T (2001) Prevention of earlobe keloid recurrence with postoperative corticosteroid injections vs. radiation therapy: a randomized, prospective study and review of the literature. Dermatol Surg 27: 323-324

17. Tu AH, Girotto JA, Singh N et al. (2002) Facial fractures from dog bite injuries. Plast Reconstr Surg 109: 1259-1265

18. Wolf KD (1998) Management of animal bite injuries of the face: experience with 94 patients.J Oral Maxillofac Surg 56: 838-842 